\title{
Characteristics of training and motivation of physicians working in emergency medicine
}

\author{
Gilson Soares Feitosa-Filho ${ }^{1 *}$, Marcelo Kirschbaum² ${ }^{2}$ Yuri Costa Sarno Neves², Bruna Melo Coelho Loureiro³, \\ Victor Augusto Camarinha de Castro Lima², Rafael Marques Calazans ${ }^{2}$, Camila Kruschewsky Falcão ${ }^{3}$, \\ Renata Trindade El Fahl ${ }^{3}$, Bianca Recarey Barreto ${ }^{3}$ \\ ${ }^{1} \mathrm{MD}$, PhD, Escola Bahiana de Medicina e Saúde Pública, Hospital Santa Izabel, Santa Casa de Misericórdia da Bahia, Salvador, BA, Brazil \\ ${ }^{2} \mathrm{MD}$, Universidade Federal da Bahia (UFBA), Salvador, BA, Brazil \\ ${ }^{3} \mathrm{MD}$, Escola Bahiana de Medicina e Saúde Pública, Salvador, BA, Brazi
}

Study conducted at Escola Bahiana de Medicina e Saúde Pública, Salvador, BA, Brazil

Article received: $6 / 30 / 2016$ Accepted for publication: 7/9/2016

*Correspondence:

Hospital Santa Izabe

Coordenação de Ensino

Address: Praça Conselheiro Almeida

Couto, 500, Nazaré

Salvador, BA - Brazil

Postal code: 40050-410

gilsonfeitosafilho@yahoo.com.br

http://dx.doi.org/10.1590/1806-9282.63.02.112

\section{SUMmARY}

Introduction: Emergency medicine is an area in which correct decisions often need to be made fast, thus requiring a well-prepared medical team. There is little information regarding the profile of physicians working at emergency departments in Brazil.

Objective: To describe general characteristics of training and motivation of physicians working in the emergency departments of medium and large hospitals in Salvador, Brazil.

Method: A cross-sectional study with standardized interviews applied to physicians who work in emergency units in 25 medium and large hospitals in Salvador. At least $75 \%$ of the professionals at each hospital were interviewed. One hospital refused to participate in the study.

Results: A total of 659 physicians were interviewed, with a median age of 34 years (interquartile interval: 29-44 years), 329 (49.9\%) were female and 96 (14.6\%) were medical residents working at off hours. The percentage of physicians who had been trained with Basic Life Support, Advanced Cardiovascular Life Support and Advanced Trauma Life Support courses was 5.2, 18.4 and 11.0\%, respectively, with a greater frequency of Advanced Cardiovascular Life Support training among younger individuals $(23.6 \%$ versus $13.9 \%$; $<<0.001)$. Thirteen percent said they were completely satisfied with the activity, while $81.3 \%$ expressed a desire to stop working in emergency units in the next 15 years, mentioning stress levels as the main reason. Conclusion: The physicians interviewed had taken few emergency immersion courses. A low motivational level was registered in physicians who work in the emergency departments of medium and large hospitals in Salvador.

Keywords: emergency medicine, physicians, motivation, clinical competence, advanced cardiovascular life support, Brazil.

\section{INTRODUCTION}

Emergency medical care is designed to intercede in situations requiring quick or immediate decisions to reduce morbidity and mortality related to acute repercussions of systemic diseases or trauma. ${ }^{1,2}$ For successful intervention in such situations, a qualified medical team, made up of professionals who are able to make quick decisions and apply effective trauma management and resuscitation techniques, is necessary. ${ }^{3}$
Care provided by the medical staff at the emergency department is a result of professional experience combined with a number of factors, namely proper training at an undergraduate or residency program, completion of immersion courses such as Basic and Advanced Cardiovascular Life Support (BLS and ACLS), motivation and perceived valorization. ${ }^{4,5}$

There is no information regarding these characteristics among emergency physicians in Brazil. This study 
aimed to define the profile of doctors working in emergency departments of medium and large hospitals in a big Brazilian city in regard to professional training, motivation and expectations towards their work setting.

\section{Method}

Study design

This was a cross-sectional study in which the primary objective was to determine the professional profile of emergency physicians working in all medium and large hospitals in the city of Salvador, Bahia, Brazil. Data were obtained through interviews (questionnaire appliance), carried out between January and March 2012. The study was approved by the Institutional Ethics Committee of Santa Izabel Hospital.

\section{Selection of participants}

A questionnaire was applied to physicians working in the emergency services of all 25 medium and large size hospitals in the city of Salvador, the third largest city in Brazil. ${ }^{6}$ Hospitals were classified according to the document "Concepts and Definitions in Health", according to which a medium sized hospital has 50 to 150 beds and a large one has 150 or more beds. ${ }^{7}$ The number of hospital beds in every institution can be found at the National Database of Health Establishments. ${ }^{8}$ All of the physicians gave consent to participate in this study. The Ethics Committee from each hospital was informed about this study. Only one institution refused to participate. Each institution provided a list with the names of all the physicians who were working in its emergency department and visits were made to each service to carry out interviews. The goal was to interview at least $3 / 4$ of the emergency medical staff of each hospital, based on the time scales provided by the coordinators of the emergency services.

\section{Data collection}

The questionnaire applied was developed by the authors for the purpose of this research and included questions concerning medical education, graduate degrees and complementary courses, experience in emergency during and after the medical undergraduate program, dedication, and job satisfaction. The median of additional years doctors intended to work in emergency services was a quantitative variable. Interviews were carried out at the emergency department of each hospital by medical students previously trained to apply the questionnaire.

\section{Primary data analysis}

Data were expressed as means and standard deviations with Gaussian distribution, or median and interquartile interval when the distribution was not Gaussian. Frequencies were reported as absolute values and percentages. Chi-square test was used for categorical variables and the Mann-Whitney and Kruskal-Wallis for continuous variables. Data analysis was performed with SPSS software, version 13.0.

\section{RESULTS}

A total of 659 physicians on duty at emergency departments of large and medium hospitals in Salvador were interviewed. This total corresponded to approximately $75 \%$ of the emergency physicians' estimate. We evaluated 24 hospitals, of which 11 (45.8\%) were public, 6 (25\%) were private, and 7 (29.2\%) had both public and private care services (Table 1). Only one hospital refused to participate. All physicians evaluated gave consent to participation in the study.

The emergency physicians were usually young, presenting a median age of 34 years, ranging from 24 to 73 years old. There was no gender predominance. The median of working hours per week in emergency shifts was 24 hours (interquartile range [IQR]: 20-42 hours).

Most physicians (64.5\%) considered they had good experience with emergency during the undergraduate program. Ninety-six physicians (14.6\%) were medical residents working at residency off hours. About half declared to have specialist qualifications (Table 1).

The percentage of doctors who had already gone through BLS, ACLS and Advanced Trauma Life Support (ATLS) programs in the past two years was 5.2\%; 18.4\%; $11.0 \%$, respectively (Table 2 ). ACLS training was the most frequent among the youngest physicians $(\mathrm{p}=0.001)$ (Table 3). When asked about what was the main reason for choosing the emergency department as their workplace, the majority chose "I like the activity" out of five alternatives (41.7\%) (Table 4). Only $13.1 \%$ were fully satisfied with their job (Table 5) and $81.3 \%$ said they intended to stop working at the emergency department in the next 15 years, pointing out "excessive stress at work" as their main reason (Table 6). The median of additional years doctors intended to work in emergency services was 5 years (IQR: 2-8 years).

In relation to their self-evaluation as emergency physicians, $11.7 \%$ claimed they performed excellently. There was a higher frequency of physicians who considered their performance excellent in the group of doctors who had completed a residency program $(14.2 \%$ against $1.6 \%$, in the non-residency group; $\mathrm{p}<0.001)$. Among the physicians satisfied with their job, 20.9\% rated their performance as excellent, against $14.4 \%$ in the group of physicians dissatisfied with their job $(\mathrm{p}<0.001)$. 
TABLE 1 General characteristics of the emergency services and interviewed physicians.

\begin{tabular}{|c|c|c|c|c|}
\hline Type of funding & Number of emergencies & \multicolumn{3}{|c|}{ Number of emergency physicians } \\
\hline Public & $11(45.8 \%)$ & \multicolumn{2}{|c|}{$332(50.4 \%)$} & \\
\hline Private & $6(25 \%)$ & \multicolumn{2}{|c|}{$172(26.1 \%)$} & \\
\hline Public and private & $7(29.2 \%)$ & \multicolumn{2}{|c|}{$155(23.5 \%)$} & \\
\hline Total & 24 & \multicolumn{2}{|c|}{659} & \\
\hline General data & $\mathrm{N} /$ frequency & Minimum & Maximum & Mean (years) \\
\hline Age & 656 & 24 & 73 & 37.7 \\
\hline Time after degree & 656 & 0 & 42 & 12.4 \\
\hline Weekly hours in the ER & 524 & 0 & 15 & 4.9 \\
\hline Specialist title: Yes & $333(50.7 \%)$ & & & \\
\hline No & $324(49.3 \%)$ & & & \\
\hline Master's degree & $33(5 \%)$ & & & \\
\hline Doctoral degree & $8(1.2 \%)$ & & & \\
\hline Sex: Male & $330(50.1 \%)$ & & & \\
\hline Female & $329(49.9 \%)$ & & & \\
\hline \multicolumn{5}{|c|}{ Completed Medical Residency Program } \\
\hline Yes (one or more) & $502(76.2 \%)$ & & & \\
\hline Internal Medicine & 97 & & & \\
\hline Cardiology & 32 & & & \\
\hline General Surgery & 110 & & & \\
\hline Pediatric & 95 & & & \\
\hline Gynecology-Obstetrics & 82 & & & \\
\hline Other residency & 235 & & & \\
\hline No residency & $157(23.8)$ & & & \\
\hline \multicolumn{5}{|l|}{ Currently resident } \\
\hline Yes & $96(14.6 \%)$ & & & \\
\hline Internal Medicine & 15 & & & \\
\hline Cardiology & 6 & & & \\
\hline General Surgery & 8 & & & \\
\hline Pediatric & 10 & & & \\
\hline Gynecology-Obstetrics & 8 & & & \\
\hline Other residency & 49 & & & \\
\hline No resident & $563(85.4 \%)$ & & & \\
\hline
\end{tabular}

TABLE 2 Frequency of knowledge and major updating courses in emergency medicine taken in the last two years.

\begin{tabular}{ll} 
Course & $\mathbf{n}=\mathbf{6 5 9}$ \\
\hline ACLS & $121(18.4 \%)$ \\
\hline ATLS & $72(11.0 \%)$ \\
\hline BLS & $34(5.2 \%)$ \\
\hline
\end{tabular}

ACLS: Advanced Cardiovascular Life Support; ATLS: Advanced Trauma Life Support; BLS: Basic Life Support.
TABLE 3 Frequency of physicians certified in the major immersion courses in emergency medicine in the last two years by age.

\begin{tabular}{llll} 
Course & $<\mathbf{3 4}$ years $(\mathbf{n = 3 1 1 )}$ & $\mathbf{2} \mathbf{3 4}$ years $(\mathbf{n}=\mathbf{3 4 5})$ & $\mathbf{p}$-value \\
\hline ACLS & $73(23.6 \%)$ & $48(13.9 \%)$ & 0.001 \\
\hline ATLS & $37(12.0 \%)$ & $35(10.1 \%)$ & 0.456 \\
\hline BLS & $19(6.1 \%)$ & $15(4.3 \%)$ & 0.300
\end{tabular}

ACLS: Advanced Cardiovascular Life Support; ATLS: Advanced Trauma Life Support; BLS: Basic Life Support. 
TABLE 4 Main reasons stated for working in emergency services.

\begin{tabular}{ll} 
Reason & $\mathbf{n = 6 5 9}$ \\
\hline I like the activity & $270(41.7 \%)$ \\
\hline It is a job opportunity & $145(22.4 \%)$ \\
\hline Good financial income & $137(21.2 \%)$ \\
\hline I want to acquire experience & $63(9.7 \%)$ \\
\hline Other reasons & $32(4.9 \%)$ \\
\hline Did not respond & $12(1.8 \%)$ \\
\hline
\end{tabular}

TABLE 5 Degree of satisfaction of the physicians with their practice in emergency services.

\begin{tabular}{ll} 
Degree of satisfaction & $\mathbf{n}=\mathbf{6 5 9}$ \\
\hline Satisfied & $86(13.1 \%)$ \\
\hline Neutral & $473(71.8 \%)$ \\
\hline Dissatisfied & $97(14.7 \%)$ \\
\hline Did not respond & $3(0.5 \%)$ \\
\hline
\end{tabular}

TABLE 6 Main reasons for not working in emergency in the next 15 years (more than one option could be chosen).

\begin{tabular}{ll} 
Reason & $\mathbf{n = 5 3 4}$ \\
\hline Stress & $339(63.7 \%)$ \\
\hline Low remuneration & $192(36.2 \%)$ \\
\hline Inappropriate working conditions & $283(53.4 \%)$ \\
\hline
\end{tabular}

When analyzing the main reason for working in the emergency department, the frequency of the answers "It's a job opportunity" and "Good financial income" was higher in the group of emergency physicians who were dissatisfied with their job $30.4 \%$ and $35.1 \%$, respectively). On the other hand, doctors in the "satisfied" group chose "I like the emergency practice" more often (52.4\%). The differences were all statistically significant $(\mathrm{p}<0.001)$.

Among the physicians who were satisfied, $64.0 \%$ intended to stop working in emergency medicine in the next 15 years, compared to $93.8 \%$ of the dissatisfied doctors and $81.8 \%$ of those who were neutral and declared themselves neither satisfied nor dissatisfied ( $p<0.001)$. Regarding the reasons for stopping working in emergency medicine in the next 15 years, $41.8 \%$ of the satisfied, $64.2 \%$ neutral and $75.8 \%$ dissatisfied physicians reported "stress at work" ( $p<0.001)$ (Table 7).

Comparing to private and mixed services, the physicians of exclusively public emergency rooms were older, more frequently specialists, less satisfied and less trained in ACLS (Table 8).

\section{Discussion}

The aim of this study was to evaluate the general and professional profile of physicians working in emergency departments in the third largest city in Brazil. There was a predominance of young doctors working in the emergency department, similar to what Rosenbach et al. ${ }^{9}$ had described. In their report, emergency physicians were younger and less qualified than physicians from other specialties.

Although male doctors are still predominant in Brazil, ${ }^{10}$ we found a balanced gender distribution. This may reflect a transition of gender predominance that is seen in Brazil among the youngest physicians. ${ }^{10}$

Half of the physicians had finished their residency program and almost $15 \%$ of the professionals were still undergoing their residency. Furthermore, the low frequency of involvement in immersion courses is surprisingly remarkable, as the majority of the most traditional courses focus on various aspects of the emergency practice. Analyzing the results by age, we observed that younger physicians engaged in ACLS courses more often than older ones, which might reflect greater motivation for continuing learning and training among those who are initiating their professional career.

A cross-sectional study conducted between 2003 and 2004 in Salvador, Brazil, evaluated knowledge about cardiopulmonary resuscitation among emergency physicians in public and private hospitals. This report showed that 70.5 and $66.9 \%$ of the doctors had never taken ACLS or ATLS courses, respectively. ${ }^{11}$

In our study, $64 \%$ of the emergency physicians reported they had good experience in emergencies during their undergraduate program. Another study, conducted with final year medical students from the Faculty of Medical Sciences of the Rio de Janeiro State University showed that $93.7 \%$ of the students had done at least one extracurricular training course during their degree. Emergency medicine and intensive care were chosen more frequently than other areas, because students felt they needed more training in these settings despite their regular undergraduate training. ${ }^{12}$ In Israel, $65 \%$ of the students from the Hebrew University - Hadassah Faculty of Medicine considered "diagnosis and management of medical emergencies" as one of the most deficient points of their medical training. ${ }^{13}$ In Iran, on the other hand, a study involving a questionnaire answered by students from Tehran University of Medical Sciences - School of Medicine showed that $69.4 \%$ of the students classified their training in medical emergency as "good" or "very good."14

In Brazil, in contrast with different countries around the world, there are few residency training programs in 
TABLE 7 Differences regarding the reasons for not working in emergency by degree of satisfaction with the job (more than one answer could be chosen).

\begin{tabular}{lllll} 
& Fully satisfied & Partially satisfied & Dissatisfied & P-value \\
\hline Stress & $23(41.8 \%)$ & $247(64.2 \%)$ & $69(75.8 \%)$ & $<0.001$ \\
\hline Low remuneration & $5(9.1 \%)$ & $128(33.4 \%)$ & $58(63.7 \%)$ & $<0.001$ \\
\hline Inappropriate working conditions & $7(12.7 \%)$ & $201(52.5 \%)$ & $74(81.3 \%)$ & $<0.001$ \\
\hline
\end{tabular}

TABLE 8 Comparisons related to the type of emergency service.

\begin{tabular}{llll} 
& Public & Private or public and private & P-value \\
\hline Age & & & 0.004 \\
$<34$ years & $138(41.8 \%)$ & $173(53.1 \%)$ & \\
$\geq 34$ years & $192(58.2 \%)$ & $153(46.9 \%)$ & $<0.001$ \\
\hline Mean formed time (years) & 15 & 10 & $<0.001$ \\
\hline Specialist title frequency & $191(57.9 \%)$ & $142(43.4 \%)$ & 0.001 \\
\hline Degree of satisfaction & & $50(15.3 \%)$ & $244(74.8 \%)$ \\
Satisfied & $36(10.9 \%)$ & $32(9.8 \%)$ & $<0.001$ \\
Neutral & $229(69.4 \%)$ & $303(92.7 \%)$ & \\
Dissatisfied & $65(19.7 \%)$ & & \\
\hline Knows ACLS & $248(75.2 \%)$ & & \\
\hline
\end{tabular}

ACLS: Advanced Cardiovascular Life Support.

Emergency Medicine. Currently in Brazil there are only 27 residency programs in Emergency Medicine, distributed in nine states. This number falls far short compared, for example, with specialties such as Internal Medicine or General Surgery, with 287 and 356 programs in all Brazilian states, respectively. ${ }^{15}$ In the United States, there are approximately 5,000 emergency departments with 25,000 physicians practicing in these centers. Currently more than 2,700 physicians are being trained in the 132 Emergency Medicine Residency Training Programs approved by the US Accreditation Council for Graduate Medical Education (ACGME). Each year these programs award degrees to approximately 800 physicians that are eligible to become certified as Emergency Medicine specialists. Emergency Medicine has been and continues to be one of the most competitive specialties for medical student applicants. ${ }^{16}$

Despite stating that the main reason for working in the emergency room was that they liked the activity, most physicians expressed their intention to stop working in this field in the next 15 years. This fact highlights the importance to study the profile of the professionals who are working in emergency services. Few professionals seemed to have plans to pursue a career in emergency medicine, even among those fully satisfied with this practice, and only some of them reported to be satisfied with their job. Curiously, the main reasons for working in the emergency department ("good financial income" and "job opportunity") were significantly associated to dissatisfaction with the job as an emergency physician.

In a study with physicians from the American Board of Emergency Medicine (ABEM) about job satisfaction in emergency practice, $23.1 \%$ of the physicians reported an intention to stop working in emergency medicine in five years time. ${ }^{17}$ Many factors may influence the ceasing of medical emergency practice, such as the great number of patients seen per hour, the stress involved and burnout syndrome. ${ }^{18,19}$ A Canadian cross-sectional study showed that emergency physicians are emotionally exhausted, with high levels of depersonalization and relatively low levels of personal accomplishment. ${ }^{20}$

A lower level of satisfaction was associated with practice in public emergency services. A study in Turkey that assessed burnout in public versus private emergency rooms also found that in the public sector, work locations, false accusations, occupational injuries and diseases, work-related permanent disabilities, and organizational support have significantly influence in self-reported perceptions of well-being $(\mathrm{p}<0.05){ }^{21}$

There were some limitations in our study. The questionnaire was elaborated and applied by the authors of the article. However, no validated instrument about this theme was found in the medical literature to be used for the purposes of the present study. It was not possible to interview the entire population of physicians working in emergencies from Salvador. This is probably because the 
population of doctors working in emergency services changes constantly, and has a fast turnover, associated to the fact that most physicians work regularly in areas other than emergency with occasional shifts in the emergency department, without an actual employment bond in the area. Even though we used a convenience sampling, we believe that our findings likely reflect the characteristics of our target-population, since more than $75 \%$ of the emergency physicians of all large and medium size hospitals were interviewed. It was not possible to compare a group that received specific training in emergency medicine with another that did not, because there were no specific training programs in the country. Some factors such as safety at work and provision of materials for an adequate job could have been included in the questionnaire in order to assess their influence on satisfaction of emergency physicians, for example. Additionally, the alternatives in some multiple choice questions might not reflect the exact thoughts of each individual.

\section{Conclusion}

We found that the emergency physicians of large and medium-sized hospitals in the city of Salvador are relatively young, without gender predominance. They have little training in emergency immersion courses, and need to be more motivated to practice their profession fully in emergency departments. This information could be extrapolated for other countries that have emergency services working under similar conditions of funds and labor, such as other developing countries or those located in Latin America.

\section{Conflict of interest}

The authors declare no conflict of interest.

\section{References}

1. International Federation of Emergency Medicine. IFEM definition of emergency medicine. Australia; 2008 [updated 2013 Oct 08; cited 2013 Nov 22]. Available from: http://www.ifem.cc/

2. European Society for Emergency Medicine. What is emergency medicine? Belgium; 1997 [updated 2013 Oct 08; cited 2013 Nov 22]. Available from: http://www.eusem.org/
3. Canadian Medical Association. Emergency medicine profile. Ottawa; 1995 [cited 2013 Nov 29]. Available from: http://www.cma.ca/

4. American College of Emergency Physicians. Emergency physician rights and responsibilities. Irving; 2014 [cited 2013 Nov 29]. Available from: http:// www.acep.org/Clinical---Practice-Management/Emergency-Physician-Rightsand-Responsibilities/?_taxonomyid=471094.

5. American College of Emergency Physicians. Use of Short Courses in Emergency Medicine as Criteria for Privileging or Employment Emergency physician rights and responsibilities. Irving; 2014 [cited 2013 Nov 29]. Available from: http://www.acep.org/Clinical---Practice-Management/Useof-Short-Courses-in-Emergency-Medicine-as-Criteria-for-Privileging-orEmployment/?_taxonomyid=471094.

6. Instituto Brasileiro de Geografia e Estatística. Estimativas da população residente. Rio de Janeiro: Instituto Brasileiro de Geografia Estatística; 2010 [cited 2013 Nov 29]. Available from: http://www.ibge.gov.br/home/estatistica/ populacao/estimativa2011/POP2011_DOU.pdf.

7. Brasil: Ministério da Saúde. Conceitos e Definições em Saúde. Brasília: Ministério da Saúde; 1977 [cited 2013 Nov 31]. Available from: http://bvsms. saude.gov.br/bvs/publicacoes/0117 conceitos.pdf.

8. Secretaria de Atenção à Saúde. Cadastro Nacional de Estabelecimentos de Saúde. Rio de Janeiro: Ministério da Saúde; [200-?] [cited 2013 Nov 31]. Available from: http://cnes.datasus.gov.br.

9. Rosenbach ML, Harrow B, Cromwell J. A profile of emergency physicians 1984-1985: demographic characteristics, practice patterns, and income. Ann Emerg Med. 1986; 15(11):1261-7.

10. Conselho Federal de Medicina. Conselho Regional de Medicina de São Paulo. Demografia Médica no Brasil: Cenários e indicadores de distribuição. São Paulo; 2013 [cited 2013 Dec 07]. Available from: http://www.cremesp.org br/pdfs/DemografiaMedicaBrasilVol2.pdf.

11. Filgueiras-Filho NM, Bandeira AC, Delmontes T, Oliveira A, Lima Júnior AS, Cruz V, et al. [Assessment of the general knowledge of emergency physicians from the hospitals of the city of Salvador (Brazil) on the care of cardiac arrest patients]. Arq Bras Cardiol. 2006; 87(5):634-40.

12. Taquette SR, Costa-Macedo LM, Alvarenga FBF. Currículo paralelo: uma realidade na formação dos estudantes de medicina da UERJ. Rev Bras Educ Méd. 2003; 27(3):171-6.

13. Eyal L, Cohen R. Preparation for clinical practice: a survey of medical students' and graduates' perceptions of the effectiveness of their medical school curriculum. Med Teach. 2006; 28(6):162-70.

14. Jalili M, Mirzazadeh A, Azarpira A. A survey of medical students' perceptions of the quality of their medical education upon graduation. Ann Acad Med Singapore. 2008; 37(12):1012-8.

15. Ministério da Educação / Portal MEC: acesso à informação. Available from: http://portal.mec.gov.br/residencias-em-saude/residencia-medica.

16. Williams AL, Blomkalns AL, Gibler WB. Residence training in emergency medicine: 21st century. Keio J Med. 2004; 53(4):203-9.

17. Doan-Wiggins L, Zun L, Cooper MA, Meyers DL, Chen EH. Practice satisfaction, occupational stress, and attrition of emergency physicians. Wellness Task Force, Illinois College of Emergency Physicians. Acad Emerg Med. 1995; 2(6):556-63

18. Lepnurm R, Lockhart WS, Keegan D. A measure of daily distress in practice in medicine. Can J Psychiatry. 2009; 54(3):170-80.

19. LeBlanc C, Heyworht J. Emergency physicians: "burned out" or "fired up"? Can J Emerg Med. 2007; 9(2):121-3.

20. Lloyd S, Streiner D, Shannon S. Burnout, depression, life and job satisfaction among Canadian emergency physicians. J Emerg Med. 1994; 12(4):559-65.

21. Tunaligil V, Dokucu AI, Erdogan MS. Determinants of general health, work-related strain, and burnout in public versus private emergency medical technicians in Istanbul. Workplace Health Saf. 2016; 64(7):301-12. 